\title{
Subauroral mesopause temperature response to solar activity in 1999-2013
}

\author{
Ammosova Anastasiia ${ }^{1}$ \\ Yu. G. Shafer Institute Cosmophysical Research and Aeronomy SB RAS \\ 677980, Lenin av. 31, Yakutsk, Russia \\ E-mail: ammosovaamemail.ru
}

\section{Gavrilyeva Galina}

Yu. G. Shafer Institute Cosmophysical Research and Aeronomy SB RAS

677980, Lenin av. 31, Yakutsk, Russia

E-mail: gagavrilyeva@ikfia.ysn.ru

\section{Ammosov Petr}

Yu. G. Shafer Institute Cosmophysical Research and Aeronomy SB RAS

677980, Lenin av. 31, Yakutsk, Russia

E-mail: ammosovlikfia.ysn.ru

\section{Koltovskoi Igor}

Yu. G. Shafer Institute Cosmophysical Research and Aeronomy SB RAS 677980, Lenin av. 31, Yakutsk, Russia

E-mail: koltigor@mail.ru

$\mathrm{OH}(6-2)$ rotational temperature trends and solar cycle effects are studied. Observations were carried out at the Maimaga station $\left(63.04^{\circ} \mathrm{N}, 129.51^{\circ} \mathrm{E}\right)$ for the period August 1999 to March 2013. Measurements were conducted with an infrared spectrograph. The temperature was determined by P- branch ratio of the molecular of hydroxyl. The monthly average residuals of temperature after the subtraction of seasonal climatic component were used for a search for the solar component of temperature response. The dependence of temperatures on solar activity has been investigated using the Ottawa $10.7 \mathrm{~cm}$ flux as a proxy. A linear regression fitting on residual temperatures yields a solar cycle coefficient of $4.24 \mathrm{~K} / 100$ solar flux units (SFU). The cross-correlation analyses showed that changes of the residual temperature follow changes of solar activity with a 25 months delay. The temperature response at the delay of 25 months reaches $7 \mathrm{~K} / 100 \mathrm{SFU}$. The value of the temperature trend after the subtraction of seasonal and solar components inconsiderable. The effect of geomagnetic activity on the subauroral mesopause temperature was evaluated to find the possible reasons for the time lag.

35th International Cosmic Ray Conference - ICRC2017

10-20 July, 2017

Bexco, Busan, Korea

\section{${ }^{1}$ Speaker}




\section{Introduction}

The global climate change is indeed an important scientific issue. According to the World Meteorological Organization, the average global air temperature, which is a very sensitive measure of climate change, increased in the surface layer by $0.3-0.6 \mathrm{~K}$ over the past 100 years [1]. The increase during the last three decades is even greater the mean value of rate for a century. According to theoretical studies, the positive trend of temperature in the lower atmosphere should be accompanied by the negative trend of temperature in the middle and upper atmosphere [2]. The mesopause region $(80-100 \mathrm{~km})$ is one of the most dynamic regions of the Earth's atmosphere, so this region is a climatic balance indicator of the entire atmosphere. One of the available methods for tracking climatic changes in the upper atmosphere is to measure the mesopause region temperature, using the emission from the night sky.

\section{Equipment and database}

The Infrared Digital Spectrograph (IDS) includes the SP-50 diffraction spectrograph and CCD camera. The CCD detector with a thermo-electronic cooling system, which maintains temperature up to $-50{ }^{\circ} \mathrm{C}$, makes it possible to perform measurements at the Yakutsk latitude from August to May. IDS simultaneously registers P-branches of the bands of OH(6-2) (the emission height is $\sim 87 \mathrm{~km}[3]$ ) and $\mathrm{O}_{2}(0-1)$ molecular oxygen (the emission height is $\sim 95 \mathrm{~km}$ [4]). The spectrograph's angle of sight is $\sim 9^{\circ}$ and is directed westward at a zenith angle of $49^{\circ}$ (the field of view is $32 \times 13.7 \mathrm{~km}$ at a height of $87 \mathrm{~km}$ ). The instrument function has an approximate Gaussian form and a half width of $\sim 0.8 \mathrm{~nm}$. The signal-to-noise ratio is $\sim 250$. The method for estimating the rotational temperature of molecular emissions is based on the least squares fit of model spectra constructed with regard to the instrument function for different previously specified temperatures to an actually measured spectrum [5]. The model spectrum whose deviation from the actual one is less than the registration noise is considered to correspond most closely to the reality; and the rotational temperature determined based on this spectrum corresponds to the temperature at the mesopause height. During such sampling, rotational temperature values with systematic errors exceeding random ones are excluded from further processing. The estimates indicate that random errors in temperature measurements vary from 2 to $5 \mathrm{~K}$ depending on the signal-to-noise ratio. The transition probabilities calculated in [6] have been used to estimate the rotational temperature from the hydroxyl band. IDS is installed at the Maimaga optical station, which is located $130 \mathrm{~km}$ north of Yakutsk. The observations were made at night at a solar declination angle of $>9^{\circ}$. The location of the observation station makes it possible to perform measurements only from the middle of August to the middle of May since the summer mesopause is constantly sunlit at the Yakutsk latitude. The molecular band emission spectra were exposed with a time resolution of 10 min. Figure 1 shows the image of the exit slit of the SP-50 spectrograph received by the CCD camera on January 2, 2011. The figure shows the spectrum of the molecular band of the P-branch $\mathrm{OH}(6-2)$ and $\mathrm{O}_{2}(0-1)$, where each point is the average value of the column of the matrix. It can be seen that the rotational structure of the P-branch of the hydroxyl band, also the branches $\mathrm{R}$ and 
$\mathrm{P}$ of the atmospheric system of molecular oxygen band (0-1), the intensity distribution in which the rotational temperature is estimated, is well resolved.

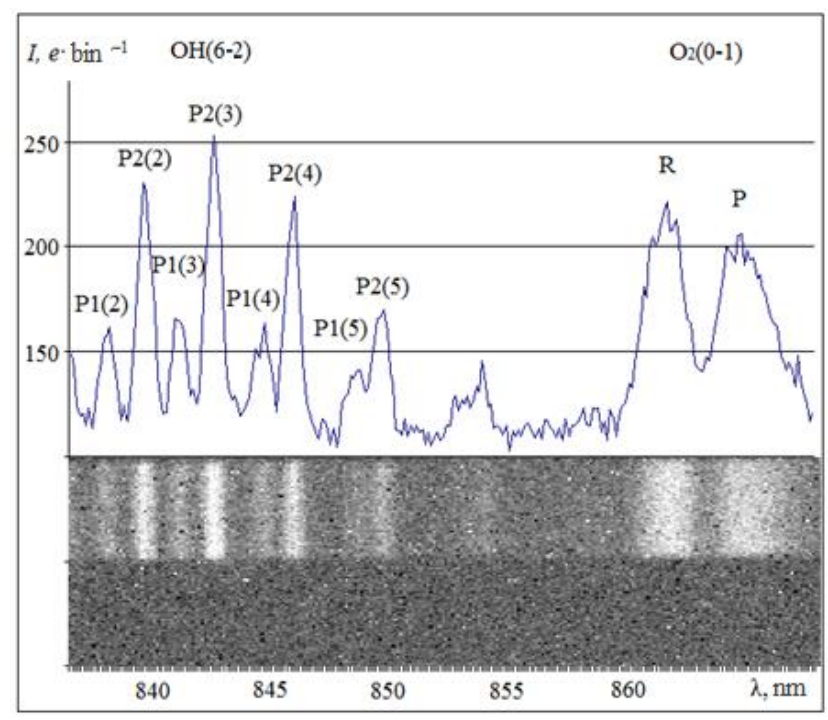

Figure 1: The contours of the $\mathrm{OH}(6-2)$ and $\mathrm{O}_{2}(0-1)$ bands spectrums and their image at the $\mathrm{CCD}$ camera output.

\section{Results}

The Maimaga rotational temperature data set comprises 2229 nightly averages which pass selection criteria. The mesopause temperature is subjected to heliogeophysical effects: seasonal change, solar radiation variations, propagation of planetary, tidal and internal gravity waves, quasi-biennial oscillations of the atmosphere, the sudden stratospheric warmings and volcanic eruptions. Tidal variation and internal gravity waves are well enough removed by using nightly mean temperatures. The seasonal change of temperature can be estimated as a sum of an annual, semiannual and terannual harmonics. If their amplitude and phases are known the season variation can easily be removed from the time series. If their amplitude and phases are known the season variation can easily be removed from the time series. The fit of annual, semiannual and terannual terms to these data with amplitudes of $28.6 \mathrm{~K}$ (maximum on day 312 ), $10.6 \mathrm{~K}$ (maximum on day 350 ) and $3 \mathrm{~K}$ (maximum on day 361 ), respectively.

In this investigation, we applied F-test to verify a goodness of the fit. Table 1 presents the values of $F$ calculated (Fcalc), and F critical (Fcrit) values for the significance level of $p=0.05$. The estimation of regression coefficient is statistically significant at Fcalc $>$ Fcrit $($ Fcrit $=3.9)$.

In Figure 2(a) and (b) monthly averaged residual temperatures and F10.7 solar flux are plotted. Figure 2(d) shows a scatter-plot of the monthly averaged residual temperatures against the monthly F10.7 value. The straight line is a best fit to the data and yields a solar response 
coefficient of $4.24 \pm 1.39 \mathrm{~K} / 100 \mathrm{SFU}$. The time series of temperatureafter subtracting the seasonal and solar components is shown in Figure 2(c).

Table 1: Regression coefficients, standard error, F-statistics and probability (p) for the linear models applied to the monthly mean residual temperatures $(\mathrm{T})$ and F10.7 in various time intervals.

\begin{tabular}{|c|c|c|c|c|c|c|c|c|}
\hline $\begin{array}{c}\text { Time interval, month } \\
\text { number from } \\
\text { January 1999 }\end{array}$ & \multicolumn{4}{|c|}{ Solar cycle response } & \multicolumn{4}{c|}{ Long-term trend } \\
\cline { 2 - 9 } & $\begin{array}{c}\text { K/100 } \\
\text { SFU }\end{array}$ & $\begin{array}{c}\text { Error, } \\
\text { K/100 } \\
\text { SFU }\end{array}$ & $F_{\text {CALC }}$ & $p$ & $\begin{array}{c}\text { K/ } / \\
\text { decade }\end{array}$ & $\begin{array}{c}\text { Error, } \\
\text { K/ } \\
\text { decade }\end{array}$ & F $_{\text {CALC }}$ & $\mathrm{p}$ \\
\hline $\begin{array}{c}\mathbf{8}<\mathbf{T}<\mathbf{1 7 3} \\
\mathbf{8}<\text { F10.7<173 }\end{array}$ & 4,24 & $\pm 1,39$ & 9,35 & 0,0027 & $-2,11$ & $\pm 1,5132$ & 1,95 & 0,1647 \\
\hline $\begin{array}{c}\mathbf{8}<\mathbf{T}<\mathbf{1 7 3} \\
\mathbf{- 1 7}<\mathbf{F 1 0 . 7}<\mathbf{1 4 8}\end{array}$ & 7,11 & $\pm 1,24$ & 33,02 & $<0,0001$ & 0,48 & $\pm 1,4076$ & 0,13 & 0,7186 \\
\hline
\end{tabular}
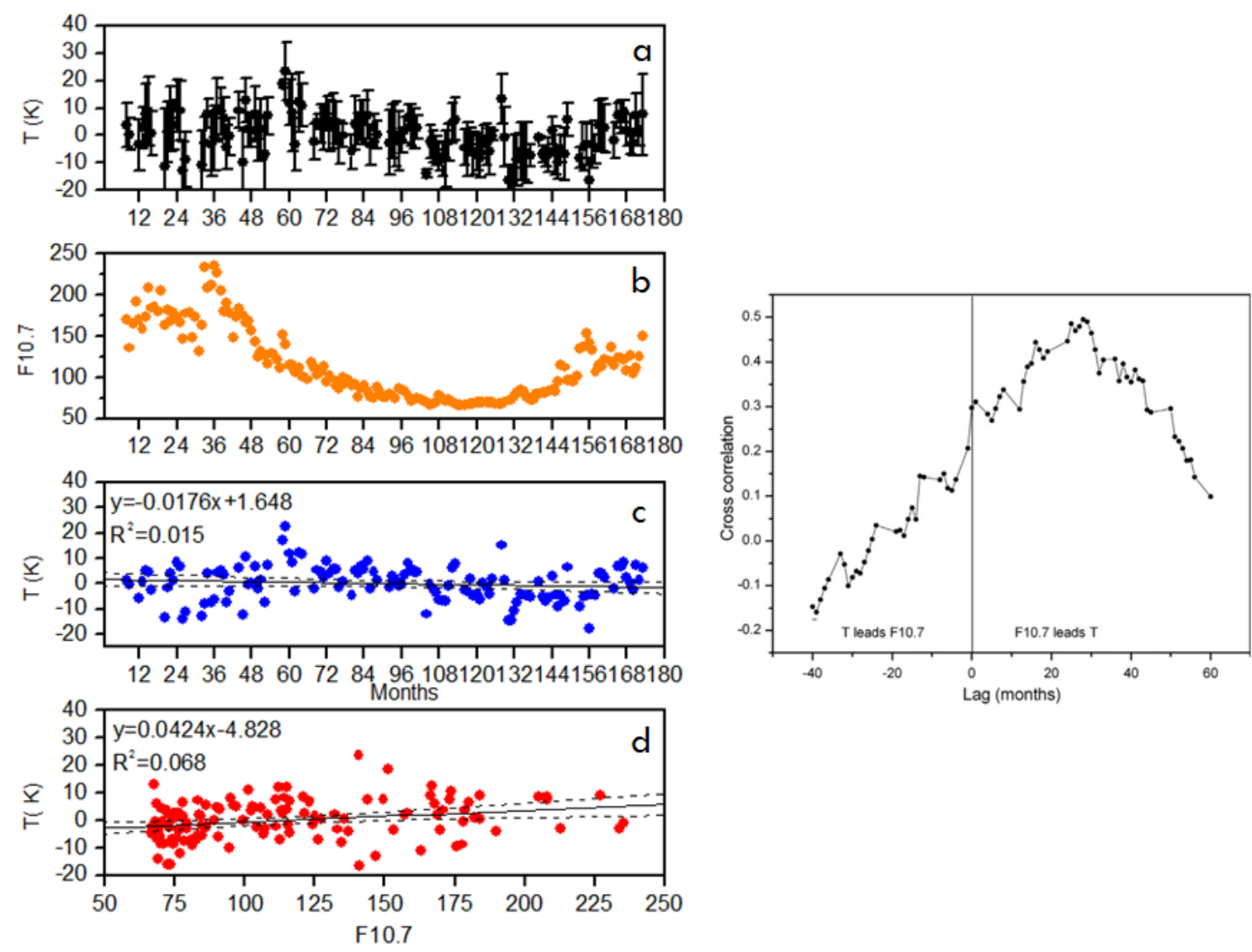

Figure 2: The monthly mean residual temperatures (a) and the monthly averaged F10.7 solar flux indexes (b) from August 1999 to March 2013. Vertical bars indicate standard errors of mean. (c) Temperature residuals after removal of both seasonal variation and solar flux effects. Straight lines are best linear fit. (d) Dependence of residual temperatures on solar flux F10.7. Linear regression curve (solid line) is shown.

Figure 3: Cross-correlation function between seasonally corrected temperatures and solar flux density F10.7 at lag $=0$. 
It is clearly seen in Figure 2(c) that after subtracting the solar components some part connected with the change of F10.7 still remains. A cross-correlation of seasonally corrected monthly temperature versus the monthly averaged F10.7 calculated with a 1-month step is shown in Figure 3. The correlation maximum is observed when F10.7 leads the temperature by 25 months. On this basis a conclusion can be made that the temperature at the level of $\mathrm{OH}$ layer responds to changes of the solar activity with a delay of approximately two years. Figure 4 shows the comparison of temperature residuals with the measurements of F10.7 since July 1997, i.e. 25 months before the beginning of regular measurements of the $\mathrm{OH}(6-2)$ band. The cross-correlation of monthly averaged temperature residuals with F10.7 is shown in Figure 5 where there is no lag between the time series. The temperature response value increases up to $7.1 \mathrm{~K} / 100 \mathrm{SFU}$
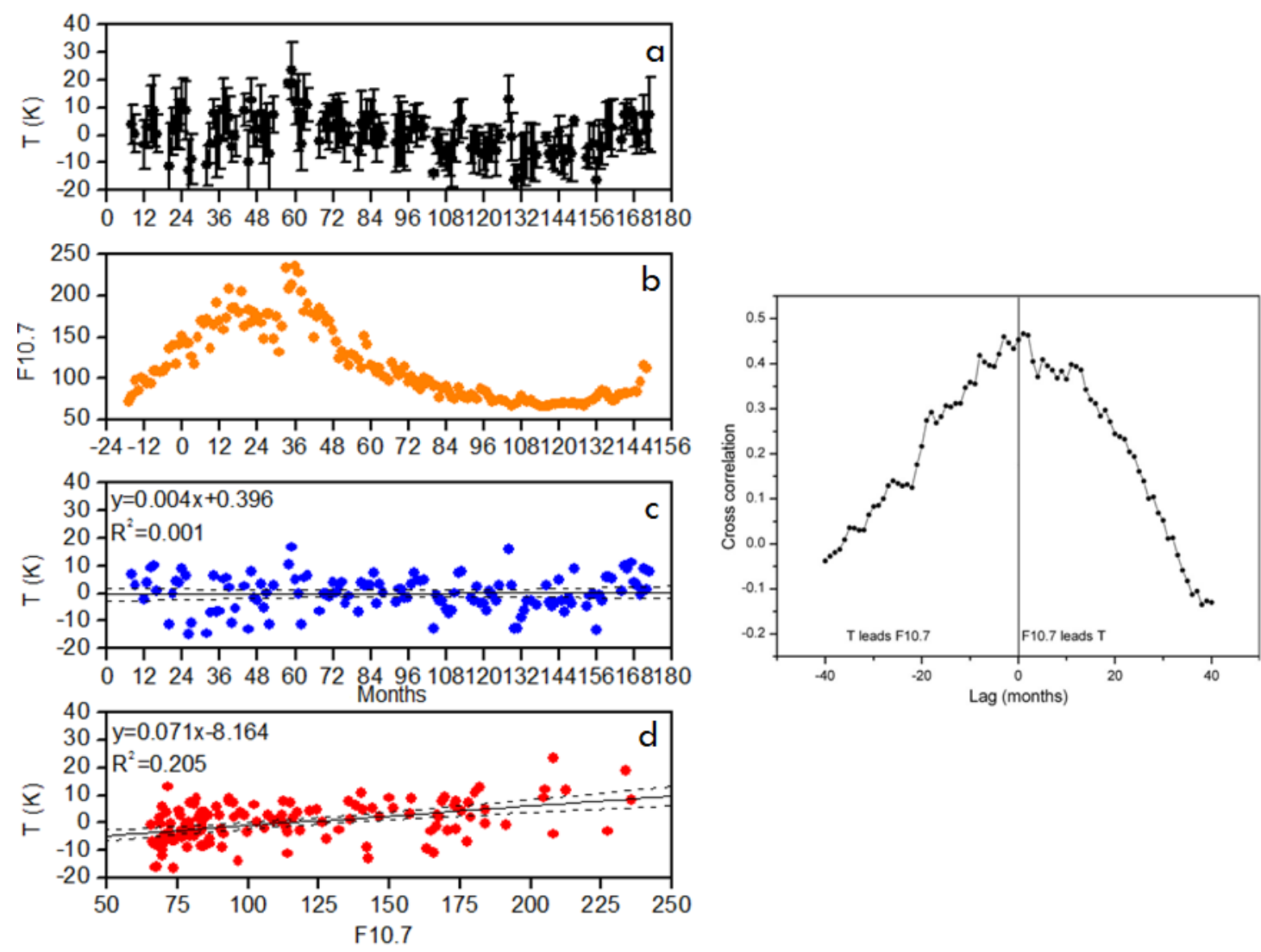

Figure 5: Cross-correlation function between

Figure 4: As in Fig. 3 but for the residual temperatures from August 1999 to March 2013 seasonally corrected temperatures and solar flux and F10.7 solar flux indexes from July 1997 to density F10.7 at lag = 25 month. April 2011.

\section{Conclusions}

The correlation analysis showed that the change of temperature is 25 months delayed relative to the F10.7 change. The comparison of seasonally corrected temperature and F10.7 without a 
shift showed that temperature response on solar activity change is equal to $4.2 \mathrm{~K} / 100 \mathrm{SFU}$ and increases up to $7 \mathrm{~K} / 100 \mathrm{SFU}$ in leading of the solar flux by 25 months. It must be taken into account that measurements of the mesopause temperature were carried out at the subauroral station, where the influence of the sun on temperature is not limited only to light radiation, it is also necessary to take into account the contribution of geomagnetic activity. The increase in the response of the mesopause temperature when shifted by 25 months relative to the solar cycle is up to $7 \mathrm{~K}$, indicates a possible connection with geomagnetic activity. It is known that geomagnetic activity reaches a maximum, approximately 2 years from the maximum of the solar cycle. All conclusions relate to the state of the upper mesosphere on subauroral latitudes.

\section{Acknowledgements}

The reported study was funded by RFBR according to the research project No. 16-35-00121 мол_а, 16-35-00204 мол_а, 17-05-00855, 15-05-05320 A

\section{References}

[1] IPCC (Intergovernmental Panel on Climate Change), Cambridge Univ. Press, 2007

[2] R.A. Akmaev, V.I. Fomichev, X. Zhu, Impact of middle atmospheric composition changes on greenhouse cooling in the upper atmosphere, J. Atmos. Solar Terr. Phys. 68: 1879-1889, 2006.

[3] D.J. Baker, A.T. Stair Rocket measurements of the altitude distribution of the hydroxyl airglow. Phys. Scr. 37( 4): 611-622, 1988.

[4] J.-H. Yee, G. Crowley, R.G.Roble, W.R. Skinner, M.D. Burrage, P.B. Hays, Global simulations and observations of $\mathrm{OI}(1 \mathrm{~S}), \mathrm{O} 2(\mathrm{blR})$, and $\mathrm{OH}$ mesospheric nightglow emissions, J. Geophys. Res. 102: 19949-19968, 1997.

[5] P.P. Ammosov, G.A. Gavrilyeva, Infrared digital spectrograph for hydroxyl rotational temperature measurements, Instrum. Exp. Tech. 43:792-797, 2000.

[6] F.H. Mies, Calculated vibrational transition probabilities of $\mathrm{OH}(X 2 \mathrm{~A})$, J. Mol. Spectrosc. 53:150180,1974

[7] Ammosov, G. Gavrilyeva, A. Ammosova, I. Koltovskoi, Response of the mesopause temperatures to solar activity over Yakutia in 1999-2013, Adv. Space Res. 54: 2518-2524, 2014. 